\title{
Effects of pulse ultrasound and kneading massage in managing individual with incessant pain at lower region of back using random allocation
}

\author{
Adesola Ojo Ojoawo ${ }^{1, *}$, Elijah Oluwatobiloba Malomo', Emmanuel Olufemi Olusegun², B. Matthew Olatokunbo Olaogun ${ }^{3}$ \\ 'Department of Medical Rehabilitation, Faculty of Basic Medical Sciences, College of Health Sciences, Obafemi Awolowo University, Ile Ife, Nigeria \\ ²Department of Physiotherapy, Osun State Specialist Hospital Asubiaro, Osogbo, Nigeria \\ ${ }^{3}$ Department of Physiotherapy, Faculty of Basic Medical and Health Sciences, Bowen University, Iwo, Nigeria
}

The study examined the effect of pulsed therapeutic ultrasound in management of patients with incessant pain at lower region of the back (PWIPLB) in comparison with kneading massage (KM). Fifty PWIPLB recruited purposively were randomly assigned into ultrasound group (USG) and KM group (KMG) equally. Subjects received back extension exercises as baseline treatment. USG received pulse ultrasound (PUS) while KMG was treated using KM. The treatments were administered 2 times weekly for a 6-week period. Severity of pain (SP) and inability of patient (IoP) were evaluated at baseline, 3rd week and 6th week of treatment. Values of the variables obtained were classified and the implications were summarised. A significant change was observed in precompared with posttreatment $\mathrm{SP}(F=32.6, P=0.000)$ and loP $(F=2.5$, $P<0.021)$ in USG. A significant change was observed in precompared with posttreatment loP $(F=4.1, P<0.05)$ but not in $\mathrm{SP}(F=2.9, P<0.086)$. In the 6th week, there was a significant reduction of $S P$ in the USG relative to $S P$ in the $K M G(F=11.98, P=0.000)$, and there was improvement significantly in the loP in the KMG relative to that in the USG $(F=2.58$, $P=0.05)$. PUS may be better than KM in management of $S P$ but KM is better than PUS in loP with PWCPLB.

Keywords: Ultrasound, Kneading massage, Low back pain, Pain intensity, Disability

\section{INTRODUCTION}

Pain at low back (PLB) is a prevalent global burden, being a commonest complaints battling with $90 \%$ adults at some points in their lives and five of ten working adults, per year Lis et al. (2007). In Africa, Louw et al. (2007) reported a prevalence of 12\% among adolescents and another of $32 \%$ among adults. These findings are in tandem with worldwide challenges of diseases of PLB suggesting an increase in incidence of PLB in Africa, a challenge which should spur members of health profession into action (Louw et al., 2007). A study in southwestern Nigeria by Omokhodion (2002) concluded that four out of ten of their participants presented with pain at the low back in the last 1 year and 33 out of 100 was affected with discomfort in the lower part of lumbar region during their study.

The goals of treatment of individuals that have discomfort at lower part of the back are that such patients should be reintegrated back to their functional capacity and ability, prevent the problem from growing chronic and reoccur again (Chien and Bajwa, 2008). Among the commonly used physiotherapeutic protocol for alleviation of patients with incessant PLB (PWIPLB) though nonpharmacological is therapeutic ultrasound (TUS) (Airaksinen et al., 2006). The use of TUS can be in two modes: continuous and pulse. Continuous TUS entails delivering the TUS wave steadily, without any interruption; while pulse TUS is intermittent in nature in the course of the treatment (Johns, 2002). In the application of TUS, sound waves convert small pocket of gas within the tissues into an empty space cavities and balls of air, which results
*Corresponding author: Adesola Ojo Ojoawo

(iD https://orcid.org/0000-0002-8045-6037

Department of Medical Rehabilitation, Faculty of Basic Medical Sciences, College of Health Sciences, Obafemi Awolowo University, Ile Ife, Nigeria

Tel: +2348033567577, E-mail: aoojoawo@yahoo.com

Received: March 1, 2018 / Accepted: April 22, 2018
This is an Open Access article distributed under the terms of the Creative Commons Attribution Non-Commercial License (http://creativecommons.org/licenses/by-nc/4.0/) which permits unrestricted non-commercial use, distribution, and reproduction in any medium, provided the original work is properly cited. 
in steady sound, disturbing the cell fluid leading to small pocket of gas to throb but not bust inward; this results into minute constant flow of fluid around the throbbing thin-globe-shape air film (Chan et al., 1998). Due to processes called non-thermal changes, various activities in the cell membrane improve the permeability of vascular wall which enables the tissues to heal faster (Allen, 2006). Kumar et al. (2013) in their review documented that, in the alleviation of patients with low back pain (PWLBP), especially for a short term period, massage may be considered as a treatment option in comparison of some active ingredient with sham. Their study also reported that there were no consistency on the efficacy of massage in comparison with other treatment like manipulative therapy standard medical care, and the use acupuncture therapy.

Continuous TUS have been investigated in patients with PWLBP (Kumar et al., 2013; Ebadi et al., 2012). Pulse TUS has been reported to be useful and effective in transdermal absorption in Wistar rats using indomethacin, gold nanoparticles and dimethyl sulfoxide (Asano et al., 1997; Silveira et al., 2016). More importantly, researches have shown comparison of massage with each of relaxation of the musculature, the use of acupuncture, taking care of self, and learning (Silveira et al., 2010). In addition, TUS requires the use of electricity which is not at regular supply in many health facilities in Nigeria, but kneading massage (KM) does not require electric power or equipment. The question is, will KM produce a similar effect with pulse ultrasound (PUS) in PWLBP? This research is aimed at evaluating the efficacy of pulse TUS and KM and to compare their effects on the severity of pain (SP) and inability of PWLBP. The main hypothesis is that there will be no significant difference in the post treatment values of SP and inability of patient in PWLBP using PUS and KM.

\section{MATERIALS AND METHODS}

Patients involved in the research were those with PWLBP receiving treatment at Physiotherapy Department, Osun State Specialist Hospital, Oshogbo, Nigeria. For a patient to be recruited in to the study, there should be complaint of discomfort at lumbosacral region, not less than 90 days. Those with history of operation at the low back, report of nerve root entrapment, disease at the internal organs, fracture of vertebra bone, or bony ankyloses were excluded from the study. The design of the study was quasi-experimental design.

Among other screening procedures, spinal motion which initiated painful sensation was documented, in that any motion in the spine that produced tension and sliding of neuron will provoke pain that are not of organic origin (Shacklock, 1995). The report of $\mathrm{x}$-ray of each patient was also reviewed and none of the reports indicated osteoporosis, carcinoma, or pot disease.

A sample size equation to compare two means according to Eng (2003) was used to ascertain how many patients would be involved for the study:

$$
\mathrm{N}=4 \delta^{2}\left(\mathrm{Z}_{\text {crit }}+\mathrm{Z}_{\text {power }}\right)^{2} / \mathrm{D}^{2}
$$

$\mathrm{N}$ is sample size for the two groups. Standard deviation is $\delta$ which could be six and the same for the two groups. $Z_{\text {crit }}$ is normal deviation that is standard which is equivalent to selective significant level (i.e., $0.05[95 \%=1.960])$. $Z_{\text {power }}$ is a deviation accepted to be excellent which is in conformity with selective strength of the statistics (i.e., $0.80=0.842$ ). $\mathrm{D}$ is the least significant change between two mean values, to be significant the value should be 2 , therefore $\mathrm{D}=5$ is chosen.

$$
\mathrm{N}=4 \times 6^{2}(1.96+0.842)^{2} / 5^{2}=45.22=45
$$

Therefore, the total number of 50 subjects were enrolled for the study: 25 subjects for the PUS group and 25 subjects for the KM group in order to give room for those that may drop out. The patient flow chat was shown in Fig. 1.

For the research, the instruments used were as follows: an Ultrasound (US) machine, a questionnaire on disability designed by Roland and Morris and a scale that measures pain by expression.

A scale that measures pain by expression is called verbal rating scale (VRS) is a 10-point scale with 1 and 10 indicating the extremes used to assess pain intensity. The pain rating scale using verbal was confirmed to be truthful in measuring pain when it was compared with another pain rating scale with vision by Williamson and Hoggart (2005). They concluded that VRS can be used interchangeably with visual analogue scale scores during the evaluation of pain in chronic stage. VRS was used to measure present pain, i.e., pain at the time of study.

The questionnaire on disability designed by Roland and Morris (1983) with acronym Rolland Morris disability Questionnaire (RMDQ) which has 24 questions is one of the tools used frequently to measure disability resulting especially from pain in the lower spinal region resulting from intervention. The RMDQ is simply evaluated by summing up the items identified by the patient, this which represent the total disability for such patient the obtainable value is 24 . One of the best outcome especially for a short term is RMDQ mainly for an individuals with a complain of pain at low back (Froud et al., 2015).

US machine (with pulse and continuous mode; Sonopuls 490, Enraf-Nonius B.V., Rotterdam, The Netherlands): This was used 


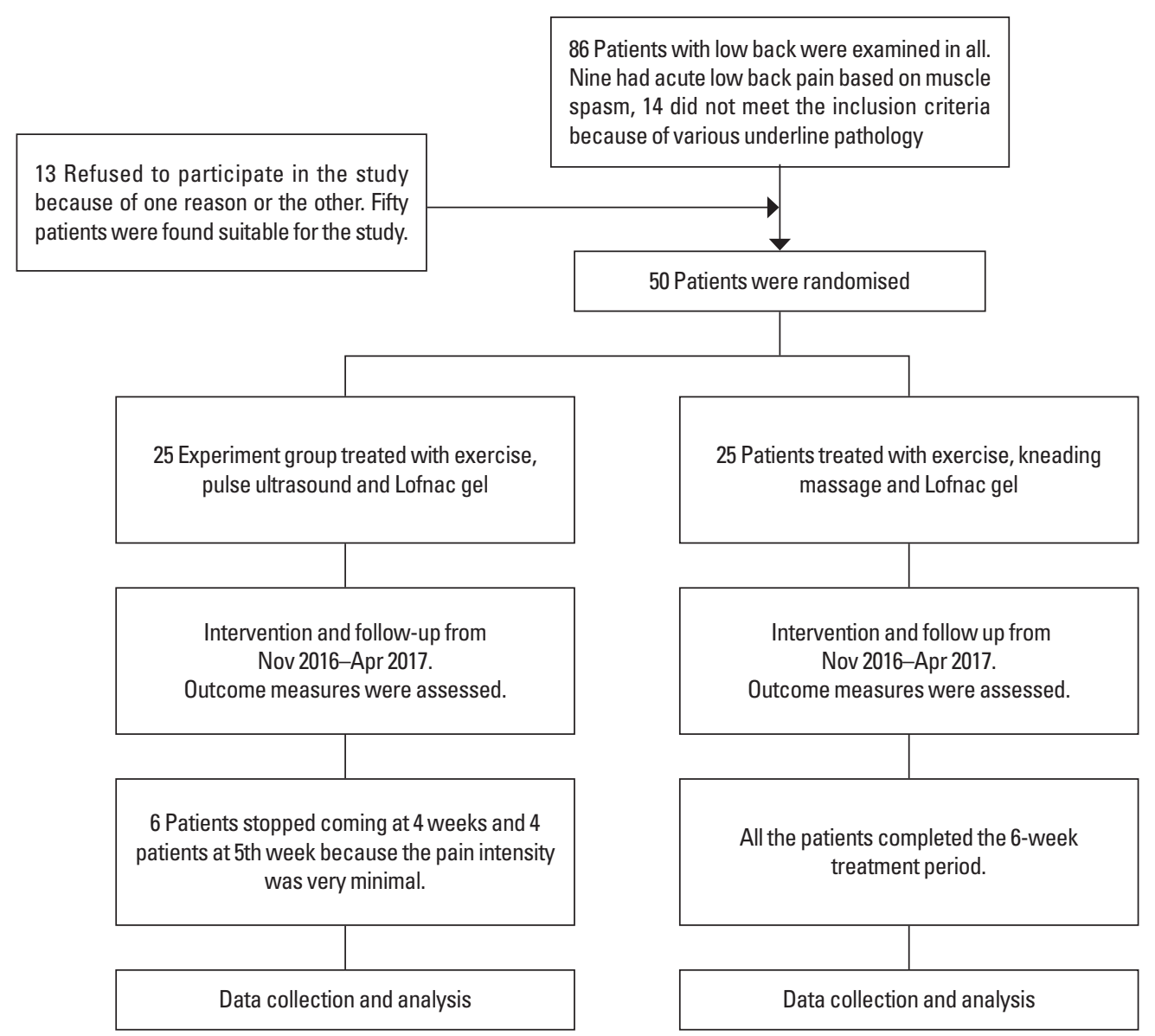

Fig. 1. Consort diagram of random allocation of subjects in two groups.

to produce the ultrasonic wave for phonophoresis.

Subjects were randomized into the two groups as follows: Envelopes which contained alphabets A and B were made. Subjects were asked to pick from the envelopes. All subjects who picked A were assigned to US (PUS) group, whereas subjects who picked B were assigned to $\mathrm{KM}$ group.

The committee on Health Research and Ethics situated at the College of Health Sciences in Obafemi Awolowo University approved the study (HREC No: IPHOAU/12/784), also all patients consented to participate in the study.

Patients in each group received 12 sessions of treatment within a period of 6 weeks of treatments. Subjects in each group were placed on stabilization exercise for $20 \mathrm{~min}$. PUS was administered for the subjects in PUS group and KM for the control group. Lofnac gel was used as topical gel for the subjects in the two groups. Treatment was administered twice (Monday and Fridays of every week for 6 weeks). Assessment was done before the treatment began and every Mondays of the week of the treatment.
The PUS group received pulse mode of US (sonoplus 490s), with mode of one MHZ and $1.5 \mathrm{w} / \mathrm{cm}$ intensity according to Ebadi et al. (2012). Grey's formula was used to estimate the duration of US for each patient (Grey, 2003). Time for the exposure is calculated as follows: for $1 \mathrm{~min}$ exposure and of radiating area of 5 $\mathrm{cm}^{2}$ of a transducer head; an area of pain at the low back of patient with $40 \mathrm{~cm}^{2}$ will need a total treatment time: The area of low back divided by the transducer head, multiply by 1 min, using the value stated, it would be $8 \mathrm{~min}$ for this study. US was used by placing the electrode on the painful area at lower region of the spine while patient lye prone and moving it slowly in circular form, Lofnac gel was used as coupling medium.

$\mathrm{KM}$ was done with the two hands maintaining a slow circular compression of soft tissues against underlying bone. Pressure was applied as the hands moved proximally, continuously maintaining a contact with the skin, according to Goats (1994). Lofnac gel was used as a coupling medium for the massage. This was done for an approximately 10 and $12 \mathrm{~min}$. 
For stabilization exercise, a supervised exercise program was employed for each patient. According to Stevens et al. (2006), patients were asked to; tilts the pelvic posteriorly. The exercises included posterior pelvic tilts, sit up from supine lying, do bridging exercise, do exercise while the knees were flexed to $90^{\circ}$ and the two upper limbs were on the floor and the muscles of the knee and hip were stretched. Not all patients could do all the exercise at a stretch; exercises were done according to the levels of tolerance and endurance of each patient. Instruction was given to each patients to stretch and hold the muscles for 20 sec between two and three stretches times per treatment, they can release it only if it was uncomfortable. The exercise which is strengthening in nature increase as patient was getting better usually commenced with five to 10 repetitions for three times per a session (Ebadi et al., 2012).

In order to avoid cointeraction, within the period of intervention and period of observation subjects were advised not to undergo any form of treatment inform of exercise. Also they were informed not to take any analgesic drug during the period of this treatment without the consent of the researchers. However, no patients requested for additional medication apart from the treatment in the department. The primary measure was SP and patients' disability was the other assessment. They were measured by a separate physiotherapist who was independent of the study every week of the treatment. Data for pretreatment, third week, and six week of treatment were used for data analysis.

The data was studied closely with package of statistical designed for SPSS ver. 17 (SPSS Inc., Chicago, IL, USA). Anthropometric indices of subjects in PUS groups were compared with that of KM using independent $t$-test. Analysis of variance (ANOVA) of Repeated measure was employed in order to ascertain the dif-

Table 1. Summary of repeated measure analysis of variance comparing the mean value of pain intensity, disability index, pretreatments, 3rd week, and 6th week of treatment in pulse ultrasound group $(n=25)$

\begin{tabular}{lccc}
\hline Variable & Mean \pm SD & $F$ & $P$-value \\
\hline Pain intensity & & 32.6 & $0.000^{* *}$ \\
Pre & $5.2 \pm 0.45$ & & \\
3rd & $3.0 \pm 0.71$ & & \\
6th & $2.4 \pm 0.54$ & & $0.021^{*}$ \\
Disability index & & 2.5 & \\
Pre & $65.3 \pm 24.3$ & & \\
3rd & $49.4 \pm 17.1$ & & \\
6th & $37.5 \pm 16.4$ & & \\
\hline
\end{tabular}

$\mathrm{SD}$, standard deviation.

${ }^{*} P<0.05,{ }^{* *} P<0.01$, statistically significant. ference in the mean values of pretreatment, 3rd week, and 6th week pain intensity and disability within the group and across the group. Post hoc analyses were carried out when necessary. Alpha level of $<0.05$ was set for the study.

\section{RESULTS}

Revealed in Table 1 is the summary of the repeated measure ANOVA comparing the pain intensity and disability index of subjects in PUS group pretreatment, 3rd, and 6th week of treatment session. SP $(F=32.6, P=0.00)$ and disability $(F=2.5$, $P=0.021)$ were significant reduced when the initial values and last treatment values in the US group were compared. Shown in Table 2 is the summary of the repeated measure ANOVA comparing the pain intensity, and disability index of subjects in $\mathrm{KM}$ group, pretreatment, 3rd, and 6th week of treatment. There was no significant reduction $(F=2.9, P=0.086)$ in pain intensity between pretreatment and 6th week of treatment in US group but there was significant reduction in disability index between pretreatment and 6th week of treatment in KM group.

ANOVA with the post hoc analysis that assessed the direction of significance was reported in Table 3. There was no meaningful change $(P>0.05)$ between PUS and KM group in the pretreatment pain intensity and disability index but pain intensity of PUS group was significantly lower $(F=11.98, P<0.000)$ than that of KM group at 6th week. Similarly, the disability in the KM group was significantly $(F=4.1, P=0.038)$ lower at 6 th week compared to PUS group. The magnitude of the effect size of comparing the third and 6th weeks of PUS group and KM group of pain intensity and disability index was shown in Table 4 . The pain intensity at 3 rd week is significantly $(F=2.710, P=0.000)$

Table 2. Summary of repeated measure analysis of variance comparing the mean values of pain intensity and disability index, pretreatment, 3rd week, and 6 th week in kneading massage group $(n=25)$

\begin{tabular}{llll}
\hline Variable & Mean \pm SD & $F$ & $P$-value \\
\hline Pain intensity & & & \\
Pre & $5.7 \pm 1.3$ & & \\
3rd & $5.2 \pm 1.2$ & & 0.086 \\
6th & $4.0 \pm 0.7$ & 2.9 & \\
Disability index & & & \\
Pre & $60.7 \pm 12.7$ & & $0.038^{*}$ \\
3rd & $47.1 \pm 13.4$ & 4.1 & \\
6th & $41.7 \pm 10.5$ & & \\
\hline
\end{tabular}

SD, standard deviation.

${ }^{*} P<0.05$, statistically significant. 
Table 3. Comparison of the outcome measure across the two groups' pretreatment, 3rd week, and 6th week $(n=50)$

\begin{tabular}{|c|c|c|c|c|}
\hline Variable & Group $A(n=25)$ & Group B $(n=25)$ & $F$ & $P$-value \\
\hline \multicolumn{5}{|c|}{ Pain intensity } \\
\hline Pre & $5.2 \pm 0.45^{a)}, * *$ & $5.7 \pm 1.3^{\mathrm{a})}$ & & \\
\hline $3 r d$ & $3.0 \pm 0.71^{b)} * * *$ & $5.2 \pm 1.2^{\text {a) }}$ & & \\
\hline 6th & $2.4 \pm 0.54^{c \mid}, * *$ & $4.0 \pm 0.7^{d l}$ & 11.98 & 0.000 \\
\hline \multicolumn{5}{|c|}{ Disability index } \\
\hline Pre & $65.3 \pm 24.3^{\text {a) }}$ & $60.7 \pm 12.7^{\text {a) }}$ & & \\
\hline $3 r d$ & $49.4 \pm 17.1^{1) !}$, & $47.1 \pm 13.4^{c}$ & & \\
\hline 6 th & $37.5 \pm 16.4^{d !}$, & $41.7 \pm 10.5^{e)}$ & 2.58 & 0.05 \\
\hline
\end{tabular}

Post hoc least significant difference.

Group A, pulse ultrasound group; group B, kneading massage group.

a-e) Mean mode with the same superscript indicates no significant difference between mean. Mean mode with different superscript indicates significant difference. ${ }^{*} P<0.05,{ }^{* *} P<0.01$, statistically significant.

reduced in PUS group than in KM group, with the mean difference of 2.2 and the effect size being significant with $\eta_{\mathrm{p}}^{2}$ of 1.15 . However, there was a significant $(F=9.810, P=0.039)$ increase (4.2) in the improvement of the disability in $\mathrm{KM}$ group at sixth week compared with PUS group with significant effect size $\left(\eta_{\mathrm{p}}^{2}=\right.$ $0.15)$.

\section{DISCUSSION}

This research was aimed at examining the efficacy of pulsed US therapy (PUS) and to compare it with that of KM on SP and inability in alleviation of patient with of incessant discomfort at the low back.

The finding from the research indicated no meaningful change in the physical characteristics of those that were in the two groups. This can be interpreted to mean that those that participated in the two groups had comparable physical characteristics, an indication that the difference obtained from the study was due to the intervention, not to the variation in the physical characteristics. The study revealed that there was a meaningful decrease in SP in the subjects in PUS group when the baseline values were compared to the third and 6th week. It has to be noted that there was no meaningful change in the SP in the KM group when the pretreatment, baseline, and 6th week mean values were compared. The present study revealed that pulsed mode of US has a significant effect in driving the active ingredient of Lofnac (methyl salicylate and diclophenac) gel into the tissues in the low back more than KM, hence the decrease in SP in the PUS group. The enhancement of taking in of drug by the skin using TUS wave is called phonophoresis Asano et al. (1997). Kanikkannan et al.
Table 4. Magnitude of effect size using partial eta square for pain intensity and disability between the two groups for $3 r d$ and 6 th week $(n=50)$

\begin{tabular}{lccccccc}
\hline Variable & $\mathrm{M} 1$ & $\mathrm{M} 2$ & $\Delta \mathrm{M}$ & $\mathrm{SD} 1$ & $\mathrm{SD2}$ & $\mathrm{SD1}+\mathrm{SD2}$ & $\begin{array}{c}\text { PETA } \\
\left(\Pi^{2}\right)\end{array}$ \\
\hline $\begin{array}{l}\text { Pain intensity } \\
\text { PUS \& KM 3rd wk }\end{array}$ & 3.0 & 5.2 & 2.2 & 0.71 & 1.2 & 1.91 & $1.15^{* *}$ \\
$\quad$ PUS \& KM 6th wk & 2.4 & 4.0 & 1.6 & 0.54 & 0.7 & 1.24 & $1.29^{* *}$ \\
Disability index & & & & & & & \\
PUS \& KM 3rd wk & 49.4 & 47.1 & 2.3 & 17.1 & 13.4 & 30.5 & 0.07 \\
PUS \& KM 6th wk & 37.5 & 41.7 & 4.2 & 16.4 & 10.5 & 26.9 & $0.15^{*}$ \\
\hline
\end{tabular}

PUS, pulse ultrasound group; KM, kneading massage group; M1, mean values for PUS group; M2, mean value for KM group; $\Delta$, change in means; $S D$, standard deviation; PETA, partial eta square.

${ }^{*} P<0.05,{ }^{* *} P<0.01$, statistically significant.

(2000) documented that phonophoresis is a process in which drugs with light molecules are driven through the skin in to the tissues with energy from US. Therapeutic PUS creates a mixture of sound wave, continuous flow and disturbances of cell fluid which is very hard to delineate (Nyborg, 1982).

A physical forces of the sound waves which moves ions and small molecules from place to the other within tissues is known as acoustic (Nyborg, 1982). This is the principle of phonophoresis. Within the cells, there are different size of small organs and molecules, some are on the same sport, while others may be in the interstitial fluid floating which may be forced to move round those at the same sport. The pushing force produced by the wave create a streamline motion of fluid in the line of and The mechanical pressure and round cell membrane this is called continuous flow or streaming (Nyborg, 1982). Another effect of PUS is disturbance of cell fluid called cavitation, this means in the minute surrounding of each cell, there are accumulation of fluid which the sound wave is forcing to vibrate (Wilkin et al., 2004). The movement of the wave of the sound in the fluid around the cells there is a feature of thinning and size reduction, this enables the minutes thin globe-shaped air filled film in the tissues to increase in size and to shrink; this process may result into injury to cell structure. The process of cavitation, may be used to explain how PUS wave can drive in active ingredients of Lofnac gel (methyl salicylate and diclofenac) into the deeper tissues and vertebrae of the low back, the site of the pain.

It will be recalled that there was no meaningful changes when the baseline, 3rd, and 6th week of SP in KM group were compared. In addition, PUS group was found to have a significant reduction of pain intensity in the 6th week of treatment session compared with KM group with a significant effect size using partial eta square. This could be interpreted that the absorption of 
Lofnac gel through KM is not deep enough to reach the depth of pain in patients with PLB. Therefore this study affirmed that KM using nonsteroidal anti-inflammatory topical gel as a coupling medium may not produce a meaningful decrease in the intensity of pain of patient with nonspecific PLB.

Furthermore we discovered that a meaningful change was noted in disability, when the 3rd and 6th week means value were compared in the KM group as well as US group. However, the effect size was not significant at the 3 rd week when the two groups were compared but the 6th week of KM was better than that of PUS with a significant effect size. The significant effect of KM supported the work of Preyde (2000) revealed that massage significantly improved both the short and long-term function better than some inert treatment would. The significant increase between in the 6th week of KM than the PUS group implied that KM has more effect on the musculature of the low back than the pulse US.

KM promotes the flow of tissue fluid and causes reflex vasodilation, it decreases muscle spasm and can stretch tissues shortened by injury (Preyde, 2000). In the process of KM which involves application of alternate pressure and mobilization, muscle tension is relieved, fibres are manipulated and muscle flexibility is increased. Massage including kneading has been reported to have the potential of providing a lot of good effects to the body system which includes enhancing the blood movement, the tension of muscles are reduced and excitement of body neurons, and the good health of individual is promoted (Preyde, 2000). The inference from this is that after a period of massage, some features characterising low back pain may be affected such as increase in the range of motion and active stiffness while the tension of such patient reduces. This buttresses the point that KM decreases the inability of patient with nonspecific pain at the low back better than PUS therapy.

The study did not follow up the improvement on the patients, owing to logistic problems within the environment of the study. Most of the patients called for the follow-up could not be reached because of poor telephone service; and appointments given to them could not be honored because of the financial plight of the patients.

The study has established that PUS reduces SP and inability of patients with nonspecific PLB significantly more than KM. However $\mathrm{KM}$ can decrease inability of the patients with pain at the low back more when compared to pulsed US.

Therefore in clinical practice, a combination of pulsed US, stabilization exercises, and $\mathrm{KM}$ can be helpful in improving the pain intensity and inability of patient with nonspecific PLB.

\section{CONFLICT OF INTEREST}

No potential conflict of interest relevant to this article was reported.

\section{ACKNOWLEDGMENTS}

The writers acknowledged the Director of Physiotherapy services of the State Specialist Hospital Asubiaro, Mr Adelowokan for allowing the study to be carried out in one the Physiotherapy Departments in the Osun State. Also Mrs Hassan M. O. and other physiotherapists in the same Department were acknowledged for their support during the data collection

\section{REFERENCES}

Airaksinen O, Brox JI, Cedraschi C, Hildebrandt J, Klaber-Moffett J, Kovacs F, Mannion AF, Reis S, Staal JB, Ursin H, Zanoli G; COST B13 Working Group on Guidelines for Chronic Low Back Pain. Chapter 4. European guidelines for the management of chronic nonspecific low back pain. Eur Spine J 2006;15 Suppl 2:S192-300.

Allen RJ. Physical agents used in the management of chronic pain by physical therapists. Phys Med Rehabil Clin N Am 2006;17:315-345.

Asano J, Suisha F, Takada M, Kawasaki N, Miyazaki S. Effect of pulsed output ultrasound on the transdermal absorption of indomethacin from an ointment in rats. Biol Pharm Bull 1997;20:288-291.

Chan AK, Myrer JW, Measom GJ, Draper DO. Temperature changes in human patellar tendon in response to therapeutic ultrasound. J Athl Train 1998;33:130-135.

Chien JJ, Bajwa ZH. What is mechanical back pain and how best to treat it? Curr Pain Headache Rep 2008;12:406-411.

Ebadi S, Ansari NN, Naghdi S, Jalaei S, Sadat M, Bagheri H, Vantulder MW, Henschke N, Fallah E. The effect of continuous ultrasound on chronic non-specific low back pain: a single blind placebo-controlled randomized trial. BMC Musculoskelet Disord 2012;13:192.

Eng J. Sample size estimation: how many individuals should be studied? Radiology 2003;227:309-313.

Froud R, Ellard D, Patel S, Eldridge S, Underwood M. Primary outcome measure use in back pain trials may need radical reassessment. BMC Musculoskelet Disord 2015;16:88.

Goats GC. Massage--the scientific basis of an ancient art: Part 1. The techniques. Br J Sports Med 1994;28:149-152.

Grey K. Distribution of treatment time in physiotherapeutic application 
of ultrasound. Physiotherapy 2003;89:696-707.

Johns LD. Nonthermal effects of therapeutic ultrasound: the frequency resonance hypothesis. J Athl Train 2002;37:293-299.

Kanikkannan N, Kandimalla K, Lamba SS, Singh M. Structure-activity relationship of chemical penetration enhancers in transdermal drug delivery. Curr Med Chem 2000;7:593-608.

Kumar S, Beaton K, Hughes T. The effectiveness of massage therapy for the treatment of nonspecific low back pain: a systematic review of systematic reviews. Int J Gen Med 2013;6:733-741.

Lis AM, Black KM, Korn H, Nordin M. Association between sitting and occupational LBP. Eur Spine J 2007;16:283-298.

Louw QA, Morris LD, Grimmer-Somers K. The prevalence of low back pain in Africa: a systematic review. BMC Musculoskelet Disord 2007; 8:105.

Nyborg WL. Ultrasonic microstreaming and related phenomena. Br J Cancer Suppl 1982;5:156-160.

Omokhodion FO. Low back pain in a rural community in South West Nigeria. West Afr J Med 2002;21:87-90.

Preyde M. Effectiveness of massage therapy for subacute low-back pain: a randomized controlled trial. CMAJ 2000;162:1815-1820.
Roland M, Morris R. A study of the natural history of back pain. Part I: development of a reliable and sensitive measure of disability in lowback pain. Spine (Phila Pa 1976) 1983;8:141-144.

Shacklock M. Neurodynamics. Physiotherapy 1995;81:9-16.

Silveira PC, Victor EG, Notoya Fde S, Scheffer Dda L, Silva Ld, Cantú RB, Martínez VH, de Pinho RA, Paula MM. Effects of phonophoresis with gold nanoparticles on oxidative stress parameters in a traumatic muscle injury model. Drug Deliv 2016;23:926-932.

Silveira PC, Victor EG, Schefer D, Silva LA, Streck EL, Paula MM, Pinho RA. Effects of therapeutic pulsed ultrasound and dimethylsulfoxide (DMSO) phonophoresis on parameters of oxidative stress in traumatized muscle. Ultrasound Med Biol 2010;36:44-50.

Stevens VK, Bouche KG, Mahieu NN, Coorevits PL, Vanderstraeten GG, Danneels LA. Trunk muscle activity in healthy subjects during bridging stabilization exercises. BMC Musculoskelet Disord 2006;7:75.

Wilkin LD, Merrick MA, Kirby TE, Devor ST. Influence of therapeutic ultrasound on skeletal muscle regeneration following blunt contusion. Int J Sports Med 2004;25:73-77.

Williamson A, Hoggart B. Pain: a review of three commonly used pain rating scales. J Clin Nurs 2005;14:798-804. 